\title{
Design for Survivability: An Approach to Assured Autonomy
}

\author{
Natalia M. Alexandrov ${ }^{1}$ \\ NASA Langley Research Center, Hampton VA 23681-2199 \\ Thomas A. Ozoroski ${ }^{2}$ \\ Analytical Mechanics Associates, Inc., Hampton VA 23666
}

\begin{abstract}
Rapidly expanding unmanned air traffic includes and will continue to include noncooperative participants. Non-cooperative behavior may be due to technical failure, a lack of appropriate equipment, a careless or malicious operator. Regardless of the cause, the outcome remains: growing density of non-cooperative traffic will increase the risk of collision between unmanned vehicles and aircraft carrying humans. As a result, the degraded safety of airspace may limit access to airspace, with adverse consequences for the traveling public and the economy. Because encounters with small non-cooperative objects, such as birds or wayward drones, can happen too rapidly for an external control system to mitigate them, it is imperative that the aircraft that carry humans survive encounters with non-cooperative vehicles. To-date, design for survivability has been practiced explicitly in the military domain. Survivability against collisions in civil aviation has been limited to tolerances against bird strikes; and these tolerances have proved inadequate on occasion. The growing risk of collision with unmanned vehicles now requires the development of survivability discipline for civilian transport aircraft. The new discipline must be infused into multidisciplinary design methods, on par with traditional disciplines. In this paper, we report on a preliminary study of survivability considerations for the civil aviation domain.
\end{abstract}

\section{Nomenclature}

$P_{S} \quad=$ survivability (probability of survival)

$P_{N S} \quad=$ non-survivability (probability of non-survival)

$P_{H} \quad=$ susceptibility (probability of collision or being hit)

$P_{N S \mid H} \quad=$ vulnerability (probability of non-survival on being hit)

\section{Introduction}

$\mathrm{V}$ arious efforts are underway to ensure the safety of the air transportation system in the face of changing airspace, in particular, the eventual, and possibly imminent, presence of large numbers of highly heterogeneous vehicles, including autonomous aircraft and Unmanned Aircraft Systems (UAS). For instance, different operation domains at different altitudes may be used for UAS. Dedicated low-altitude "drone highways" in the sky have been proposed by Amazon for their Prime Air delivery ${ }^{3}$. The Federal Aviation Administration (FAA) is partnering with industry associations to promote safe use of $\mathrm{UAS}^{4}$. Geofencing is being considered for separating various domains in airspace and on the ground ${ }^{5}$.

These and other efforts will likely safeguard operations of a large portion of airspace. However, these measures rely on voluntary cooperation of all participants and a certain minimum level of functioning equipage. Whereas, arguably, the majority of the airspace participants will exhibit cooperative behavior, some autonomous participants will not. In fact, non-cooperative aircraft are already here. Drones interfering with firefighting efforts in California

\footnotetext{
${ }^{1}$ Senior Research Scientist, Mail Stop 442, AIAA Associate Fellow.

${ }^{2}$ Aerospace Engineer.

${ }^{3}$ http://www.gizmag.com/amazon-drone-highways/38669/

${ }^{4} \mathrm{http} / / /$ knowbeforeyoufly.org/

${ }^{5}$ http://www.gizmag.com/nasa-air-traffic-control-drones/36509/
} 
in July 2015 are one well-known example of non-cooperative behavior with serious consequences ${ }^{6}$. The problem of non-cooperative behavior, whether reckless or malicious or simply uninformed, will be exacerbated with growing air traffic density. The FAA had expected nearly a million UAS to be sold during the 2015 holiday season alone ${ }^{7}$. Per recent headlines, "Reports of errant drones flouting U.S. regulations including flying too close to passenger airliners and other aircraft surged late last year to an average of four incidents per day, according to FAA data" and "Drone Close Calls, Sightings by Airliners Up Fivefold".

At the time this paper is written, civilian UAS are still relatively small, light, and primitive. They fly short distances, at low altitudes, and are not robust with respect to weather. However, even light UAS have a hard component - the battery, which can present danger to such areas of a large aircraft as the fuel-bearing part of the wing. Moreover, given the low cost of technology, the size and performance of UAS will ramp up quickly. Hence, non-cooperative behavior, whether unwitting or intentional, will present an ever growing danger to aircraft carrying passengers, ${ }^{9}$ as availability of UAS and density of UAS traffic increase.

This situation necessitates a clean-slate consideration of survivability and self-preservation of a benign aircraft in the presence of dense autonomous and unmanned traffic. Traditionally, full-blown survivability has been a concern of the military domain. Civilian aircraft design has dealt with a more limited set of survivability issues, such as the impact of bird strikes, weather, fire, and mechanical failure. Danger from collisions with other aircraft has been prevented via a set of appropriate separation bounds within regulated airspace, with information and directions provided by the external air traffic control system and some on-board equipment. The changing nature of the vehicles populating the airspace means that civilian aircraft design must now explicitly include considerations of survivability in the event of collision with other vehicles.

One of the salient features of encounters with non-cooperative objects is the extremely short time line of the event. For instance, during the "Miracle-on-the-Hudson" incident (15 January 2009, US Airways Flight 1549), only 1.5 seconds elapsed between the pilots noticing the flock of birds and the bird strikes that ultimately downed the aircraft. Neither an external air traffic control system, nor the human pilots, nor (at present) the aircraft control system can take protective action at such time scales. Ensuring the safety of humans on an aircraft necessitates detecting (sensing and perception) a non-cooperative UAS automatically, making an autonomous, machine decision, and implementing it, all in time for the aircraft systems' actions to weaken the collision and mitigate its consequences.

This work deals with survivability during potential encounters of aircraft carrying humans (pilots or pilots and passengers) with non-cooperative aircraft, where collision has not been prevented by the Traffic Alert and Collision Avoidance System (TCAS) or its likely successor, the Airborne Collision Avoidance System (ACAS X), and is imminent. In the following sections, we give some preliminary observations on survivability in civil aviation; lessons learned from design for aircraft survivability in the military domain; and the applicability of these design principles to civil aviation. We then propose a path toward developing a survivability discipline for aircraft carrying humans in an airspace that includes non-cooperative participants.

\section{Preliminary Investigation}

This study started with a qualitative consideration of various aspects of vehicle operation that impact survivability. Because flocking birds present a known threat to civilian aircraft, we collected information on the physics of bird strikes, to serve as a surrogate model of non-cooperative UAS.

\section{A. Birds and Engines}

Canada geese are a common threat due to their weight (a male from 8.41 to 9.23 pounds; a female from 7.31 to 7.75 pounds), flocking behavior, attraction to grazing sites at airports, and the year-round presence of their resident populations. Only fourteen North American bird species are at least as large as a Canada goose.

Roughly $50 \%$ of all bird strikes involve the aircraft engine, and engine certifications require designing and planning for operations after bird strikes occur. A "large flocking bird" requirement was added in 2007 that stipulated that the engine must function at a specified decreased performance after ingesting a 5.0 pound bird. It has

${ }^{6}$ http://www.npr.org/2015/07/23/425654435/california-firefighters-forced-to-call-off-missions-after-drone-

interference

${ }^{7} \mathrm{http} / / /$ aviationweek.com/commercial-aviation/faa-nightmare-million-christmas-drones

${ }^{8}$ ATCA Online News, 3/28/2016

${ }^{9}$ As well as to humans on the ground; however, explicit consideration of this aspect is beyond the scope of this paper. 
since been determined that the bird-ingestion design case is not defined by the highest kinetic energy case, but rather by the lowest operating speed. At the lowest operating speed, comparatively larger pieces of a bird will cause more damage to the engine core. Such certification requirements would not address the ingestion of a heavier drone. Moreover, despite the bird ingestion requirements, birds have downed aircraft, pointing to the ad hoc nature of such requirements and the difficulty of encompassing every bird strike situation in the requirements.

\section{B. Agility and Evasive Maneuvers}

In January of 2009 a twin-engine aircraft encountered a flock of Canada geese shortly after takeoff. Power was lost in both engines and the pilot glided to a safe landing on the Hudson River. A review of the NTSB investigation and the event timeline provided detailed information about the flight. The aircraft was operating at an altitude of 2818 feet above ground level at a speed of about $246 \mathrm{mph}$ around the time of the incident. The cockpit voice recorder indicated that both pilots saw the birds, but impact occurred one second later.

$$
\begin{array}{ll}
\text { 15:27:10.4 } & \text { HOT-1 birds. } \\
\text { 15:27:11 } & \text { HOT-2 whoa. } \\
\text { 15:27:11.4 } & \text { CAM [sound of thump/thud(s) followed by shuddering } \\
\text { sound] } & \\
\text { 15:27:12 } & \text { HOT-2 oh \#. }
\end{array}
$$

Reacting to such a threat in such short time would be difficult for any piloting system, but response might be possible if sensors provided additional reaction time. A Boeing source ${ }^{10}$ warns pilots: "Below 2,000 feet, climb at the maximum rate to reduce the flight time exposure to a strike hazard" and "Avoid or minimize maneuvering at low altitudes to avoid birds". Rapid maneuverability to avoid unregulated UAS may not be acceptable as a regular occurrence on a civilian aircraft, even if proven to be structurally and mechanically feasible, due to physiological constraints on acceptability of maneuvers to passengers. Survivability in the presence of a few birds (or drones) may not require sharp maneuvering; slight changes in the path to weaken collisions with critical sections of the aircraft may suffice, if

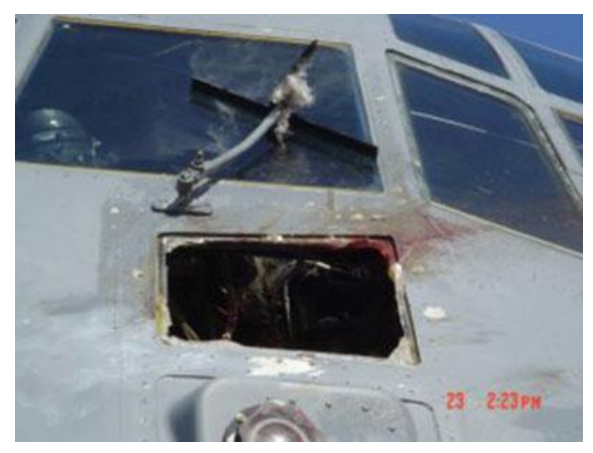

Figure 1. C-130 Strikes Bald Eagle. www.faa.gov. supported by appropriate automation. Large flocks of birds or drones present a more difficult challenge and may necessitate adding structural features and countermeasures that disable impending threats.

\section{Structural Enhancements}

According to Wikipedia, the average weight of a female bald eagle is 12 pounds. An impact of a soaring bald eagle on an airplane climbing at $200 \mathrm{mph}$ would yield the kinetic energy of a 1,000-pound weight dropped from a height of 16 feet. The aftermath of a collision between a bald eagle and a military aircraft, depicted in Figure 1, underscores that structural enhancements may not present a viable design option for even moderate rest weights of a colliding object. Although adequate structural enhancements in the form of ruggedized structure, or armor, may be prohibitive in terms of performance and cost impact, other structural measures, such as re-arrangement of critical components may be considered.

\section{Countermeasures}

Recent news (e.g., Aviation Week ${ }^{11}$ ) indicates that the FAA and other U.S. and international considerations may evaluate UAS countermeasures at airports. Such systems would include passive and active electronic countermeasures to identify and disable UAS, with possible means of taking control of the UAS and tracking the UAS owners. Lasers and mechanical devices appear in the news as well. While to-date we have not seen an announcement of a viable device for an aircraft, capable of operating at appropriate speeds, the approach is certainly plausible for an airborne countermeasure, in principle.

\section{E. Observations}

Of course, detecting danger and avoiding collisions is much preferable to attempting recovery following a collision. Therefore, detect-and-avoid capabilities are in continual development. However, given the difficulty of

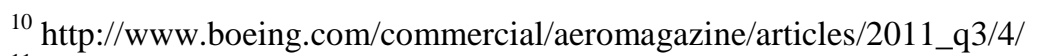

${ }^{11} \mathrm{http}: / /$ aviationweek.com/technology/counter-uas-special-report-countermeasures-options
} 
detection and avoidance under the complex conditions of a realistic and dense environment, it is impossible, in principle, to prove that detect-and-avoid capabilities will prevent a certain amount of safety degradation in airspace, at least in the foreseeable future. Therefore, an aircraft carrying passengers requires explicit survivability enhancements, to maintain passenger safety.

Aside from simply adding armor, which is the least desirable option because of the inevitable loss of performance via increased weight, every other survivability measure (maneuvers, control, component and function health monitoring, self-healing, and so on) will require the development of autonomous artificial intelligence systems, simply because humans are incapable of reacting on the timescales necessary to assess and mitigate certain types of collisions, especially those involving several threats at close range.

\section{Applicability of the Survivability Approach from the Military Domain}

Survivability in the military domain deals with combat survivability and is defined as the capability to withstand a human-made hostile environment. Vast public domain literature on military aircraft survivability provides details (see, e.g., [1-25]) of survivability components. Major differences in the physical attributes of the military and civilian aircraft (e.g., structural toughness, agility, and armament), as well as the quality of threats and dissimilar loss tolerances, do not allow for a one-to-one conversion of the military survivability techniques to civil aviation. However, there are a number of fundamental principles that can be adapted for application to civilian vehicle design and countermeasures.

\section{F. Measuring Survivability}

In an excellent reference [1], R.E. Ball, provides a probabilistic framework for defining and estimating survivability of a military aircraft. The conceptual framework can be adapted, in part, as an overall scheme for estimating civilian aircraft survivability.

Survivability is measured as the probability of survival, $P_{S}$, and is viewed as complementary to killability. Survivability and killability are mutually exclusive and form a complete (exhaustive) system in the military domain. However, killability is interpreted in the general sense that includes an inability to complete a mission. In the civilian domain, we must use a more explicit measure - non-survivability, $P_{N S}$, because the primary mission of the aircraft is to deliver humans on board to the ground safely. Not fulfilling the mission of getting to the original destination as a result of a collision is still within the bounds of survivability if the humans on board survived. Thus,

$$
P_{S}=1-P_{N S}
$$

Non-survivability depends on the susceptibility of the aircraft and its vulnerability. Susceptibility, $P_{H}$, is the probabaility of being hit. Vulnerability, $P_{N S \mid H}$, is the probability of the hit being fatal, i.e., the conditional probability of not surviving on being hit. Thus,

$$
P_{S}=1-P_{N S}=1-P_{H} P_{N S \mid H}
$$

This simply quantifies survival as either not being hit or, if hit, not crashing.

The probabilities can be further decomposed, based on specific scenarios and events. The quantification of probabilities in the military domain is done via construction of scenarios, event tree diagrams, simulations, and tests.

Improving $P_{S}$ requires reductions in susceptibility and vulnerability.

\section{Susceptibility}

Susceptibility differs significantly between the military and civilian domains. Encountering a non-cooperative drone in civilian airspace would likely be a random event, whose probability would depend on the density and attributes of traffic at specific altitudes, with rare exceptions of malicious participants. The ability to detect danger in time for evading it or employing a countermeasure, in any domain, is preferable to design-in survivability features that involve vehicle shape, weight and therefore cost. Given the probable randomness of the non-cooperative encounters in civilian airspace, a careful investigation of fundamental capabilities of the present and anticipated detection, autonomous decision making and avoidance capabilities are in order to arrive at requirements and tradeoffs for design-in features vs. operational actions and countermeasures. 


\section{Vulnerability}

Because of the randomness factor in non-cooperative encounters, arguably, vulnerability reduction should be the emphasis of survivability improvement in civil aviation.

The current approach to arriving at specific measures is complex. An aircraft is composed of a multitude of components, the vulnerability of each contributing to the vulnerability of the aircraft. Vulnerability reduction is performed via three main stages: identification of the critical components and their damage modes; vulnerability assessment; and design for low vulnerability.

The first stage is extremely complicated and depends on the detailed technical description of a specific aircraft. The second stage depends, in addition, on the properties of the threat. The final stage relies on heuristically proposed measures and trade studies to determine relative merits of the proposed solutions. Solutions are based on a number of general principles:

- Critical component redundancy with separation. E.g., multiple engines, control surfaces.

- Critical component location. Prevent cascading damage.

- Passive damage suppression. Fault tolerant design.

- Active damage suppression. E.g., fire detection and suppression.

- Critical component shielding. Shield by other components or additional structure.

- Critical component elimination. E.g., replacing the pilot with automation.

\section{G. Observations}

While many features of the approach to reducing vulnerability can be adopted for civilian aircraft use, the approach also presents a number of difficulties, as follows:

- The quantification of vulnerability appears to be based on a great deal of detailed design having been accomplished; adding survivability features appears to be an a posteriori measure. The trend in design of civilian aircraft is to increase discipline fidelity at earlier stages of design, to improve the quality of design and shorten the design cycle time. This would require for survivability to be included in the multidisciplinary design processes simultaneously with other disciplines. Thus, the quantification of survivability would require the development of physics-based models that would yield measures of vulnerability in terms of the design variables, objective and constraint functions in the design problem formulation.

- Given the need for a higher degree of assurance for survival of each civilian aircraft carrying passengers, a great deal of accuracy would be needed for uncertainty quantification and propagation in the evaluation of vulnerability, which does not appear to be present in the vulnerability estimation in the military domain.

- Given thousands of components on an aircraft, the approach to identifying critical components, damage modes and potential mitigation via discrete enumeration is likely to give rise to problems of very large dimension, whereas reducing the design space for tractability may cause missed salient failure conditions or promising designs.

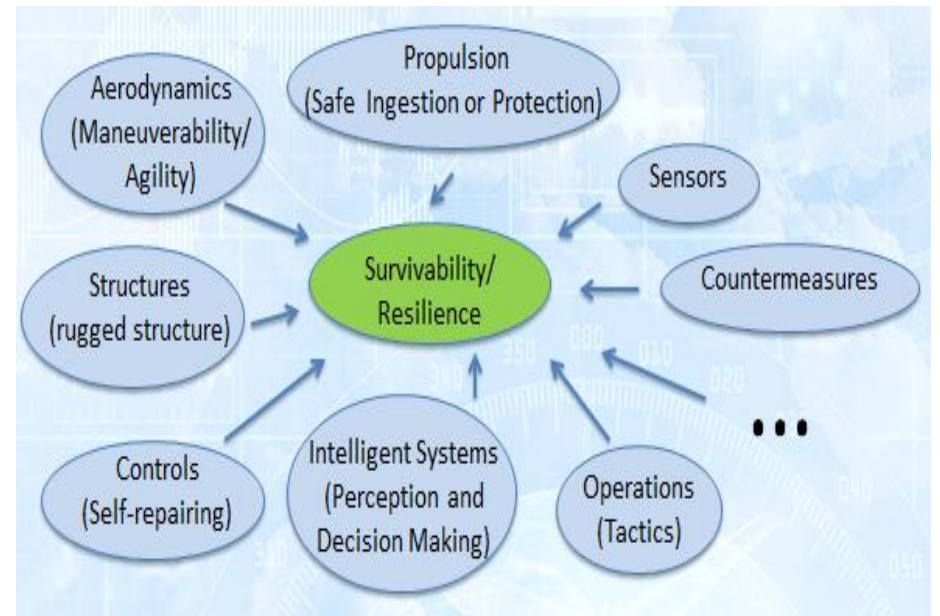

Figure 2. Some Multidisciplinary Contributions to Survivability

\section{A Quantification Path}

Survivability is affected by contributions from all disciplines. Some of the disciplines are shown in Figure 2. The list is not exhaustive. The sensory systems of the aircraft must continually monitor the external environment and internal state of the aircraft to inform the intelligent autonomous perception and decision making system, which, in turn, issues commands to the aircraft, to avoid a collision, weaken it, or recover sufficiently to deliver the passengers to the ground safely. The traditional disciplines are responsible for the actions dictated by the AI

system to be physically implementable. In order to achieve this, survivability must be directly 
connected, through variables, objectives and constraints, with all relevant disciplines in the Multidisciplinary Design Optimization (MDO) process.

We conjecture that the probabilities that comprise the definition of survivability can be decomposed all the way to the level of components or aggregates of components to allow for mapping the familiar disciplinary variables from aerodynamics, structures, control, and propulsion into models of component survivability, ultimately leading to an analysis, direct and inverse MDO formulations, with explicit accounting for uncertainties in the parameters of the problem. Once candidate problem formulations are established, sensitivity analysis should provide explicit sensitivity of survivability with respect to specific disciplinary parameters and technologies for enhancing survivability, as well as bounds of a particular discipline's contribution to survivability. This is one of the paths currently under investigation.

\section{Concluding Remarks}

"Assured Autonomy"12 is a term that embodies safe integration of UAS, autonomous systems, and systems under hybrid human-machine control into the National Airspace. Assuring autonomy involves a broad range of research and development activities aimed to ensure the safety of airspace for its users.

In assuring autonomy, it is important to address two distinct components of the technical challenge: the controllable, cooperative component and the non-cooperative component. Detect-and-avoid technologies in development for cooperative autonomous traffic should mitigate non-cooperative behavior, in large part, as well. However, this development will take time, while non-cooperative UAS are beginning to impact the safety of the airspace already. Moreover, arguably, with growing density and diversity of UAS, the detect-and-avoid technologies of the future will not contain all non-cooperative vehicles, in principle. Hence, non-cooperative traffic will increase the risk of collisions for vehicles carrying humans with autonomous unmanned vehicles, ultimately degrading the safety of airspace, with negative consequences for the traveling public and economy.

We claim that the explicit inclusion of survivability measures into aircraft design is necessary to prevent such safety degradation and to ensure access to airspace with dense autonomous traffic. The military aircraft survivability discipline is an excellent source of lessons learned for developing a survivability discipline for civil aviation. However, distinct mission goals, the different nature of aircraft design, the complexity of the processes and the preponderance of heuristic procedures make direct use of military survivability techniques not entirely applicable in the civilian domain. We are cautiously optimistic that our current work, which includes derivation of the analysis and design problems for survivability, will lay a foundation for a rigorous framework for infusing survivability into multidisciplinary design of civilian aircraft.

\section{Acknowledgments}

The authors would like to thank the Incubation element of the NASA Convergent Aerospace Solutions (CAS) Program for supporting this work during the period 10/01/2015-09/30/2016.

\section{References}

[1] R. E. Ball, The Fundamentals of Aircraft Combat Survivability Analysis and Design, Second Edition, AIAA Education Series, 2003

[2] L. M. Nicolai, G. E. Carichner, Fundamentals of Aircraft and Airship Design, AIAA Education Series, 2010

[3] D. B. Atkinson, P. Blatt, L. Mahood, D. W. Voyls, Design of Fighter Aircraft for Combat Survivability. Sae Transactions. 78, 159-\& (1969).

[4] J. O. Berger, Wald, Abraham Work on Aircraft Survivability - Comment. Journal of the American Statistical Association. 79, 267-269 (1984).

[5] D. Brown, C. Miller, M. Schumacher, K. Werner, S. T. Crino, Improving Special Operations Aircraft Survivability through Better Ballistic Protection Systems , 2009), pp. 158.

[6] J. Clements, R. Robinson, L. Bunt, J. Robinson, Missile Airframe Simulation Testbed - MANPADS (MAST-M) for Test and Evaluation of Aircraft Survivability Equipment. Technologies for Synthetic Environments: Hardware-in-the-Loop Xvi. 8015, 80150A (2011).

[7] S. C. Colwell, A Cost-Effective Method to Determine Ground Survivability of Strategic Aircraft. Oper. Res. S 14, B203$\&(1966)$.

[8] A. H. Cooper, Survivability of Military Vstol Transport Aircraft. Sae Transactions. 78, 159-\& (1969).

${ }^{12} \mathrm{http}: / /$ www.aeronautics.nasa.gov/strategic-plan.htm 
[9] L. Jia, Z. Tong, C. Wang, S. Li, Aircraft Combat Survivability Calculation Based on Combination Weighting and Multiattribute Intelligent Grey Target Decision Model. Mathematical Problems in Engineering. 8934749 (2016).

[10] J. Kang et al., Aircraft Survivability and Sensors Alignment Techniques. Journal of the Korean Society for Aeronautical Science and Flight Operation. 16, 29-36 (2008).

[11] F. Leary, Tactical Aircraft Survivability. Space Aeronautics. 47, 68-\& (1967).

[12] K. Lee, et al. Aircraft Combat Survivability Analysis based on the Random Variable Weighted Score Algorithm. Journal of the Korean Society Aeronautical and Space Sciences. 41, 883-890 (2013).

[13] M. Mangel, F. J. Samaniego, A. Wald, Work on Aircraft Survivability. Journal of the American Statistical Association. 79, 259-267 (1984).

[14] M. Mangel, F. J. Samaniego, A. Wald, Work on Aircraft Survivability - Rejoinder. Journal of the American Statistical Association. 79, 270-271 (1984).

[15] G. I. Nesterenko, Service life and survivability of aircraft structures. Materials Science. 32, 166-178 (1996).

[16] S. Noh, C. Choi, Predicting the Operational Effectiveness of Aircraft Survivability Equipment Suite. International Journal of Engineering and Technology. 4, 372 (2012).

[17] V. S. Safronov, S. E. Zaytsev, M. Y. Kalajgin, Estimation Of Survivability Of The Aircraft Wing Design Taking Into Account Damage Accumulation. Polet.Obshcherossiiskii Nauchno-Tekhnicheskii Zhurnal., 46-54 (2012).

[18] V. S. Safronov, S. E. Zaytsev, M. Y. Kaljagin, Optimal Design Console Wing Aircraft With The Requirement Of Survivability Of The State Aviation. Polet.Obshcherossiiskii Nauchno-Tekhnicheskii Zhurnal., 40-50 (2013).

[19] V. S. Safronov, Accounting Requirements for the Design Survivability of Shell Structures Aircraft. Aviakosmicheskoe Priborostroenie., 17-25 (2015).

[20] V. S. Safronov, Accounting Requirements for the Design Survivability of Flat Panel Aircraft. Aviakosmicheskoe Priborostroenie., 55-60 (2015).

[21] R. Soannini, P. Froment, Survivability Increase of Military Transport Aircraft. Nouvelle Revue Aeronautique Astronautique., 44-52 (1995).

[22] L. Throndson, Combat Survivability with Advanced Aircraft Propulsion Development. J. Aircr. 19, 915-920 (1982).

[23] J. M. Vice, Survivability Vulnerability Information Analysis Center (Surviac) - a Tool for the Aircraft Survivability Community. J. Aircr. 24, 511-515 (1987).

[24] H. Wang, S. Li, N. Chen, Simulation and Analysis of Aircraft Survivability Model the Impacted by Rain. Fire Control \& Command Control. 8, 020 (2012).

[25] X. Wang, B. Song, Y. Hu, Analytic Model for Aircraft Survivability Assessment of a One-on-One Engagement. J. Aircr. 46, 223-229 (2009). 